Astrophys. J., Submitted

Preprint typeset using $\mathrm{LAT}_{\mathrm{E}} \mathrm{X}$ style emulateapj v. 5/2/11

\title{
LOW-MASS SUPPRESSION OF THE SATELLITE LUMINOSITY FUNCTION DUE TO THE SUPERSONIC BARYON-COLD-DARK-MATTER RELATIVE VELOCITY
}

\author{
Jo BOvY ${ }^{1}$ AND CORA Dvorkin \\ Institute for Advanced Study, Einstein Drive, Princeton, NJ 08540, USA \\ Astrophys. J., submitted
}

\begin{abstract}
We study the effect of the supersonic baryon-CDM flow, which has recently been shown to have a large effect on structure formation during the dark ages $10 \lesssim z \lesssim 1000$, on the abundance of luminous, low-mass satellite galaxies around galaxies like the Milky Way. As the supersonic baryon-CDM flow significantly suppresses both the number of halos formed and the amount of baryons accreted onto such halos of masses $10^{6}<M_{\text {halo }} / M_{\odot}<10^{8}$ at $z \gtrsim 10$, a large effect results on the stellar luminosity function before reionization. As halos of these masses are believed to have very little star formation after reionization due to the effects of photo-heating by the ultraviolet background, this effect persists to the present day. We calculate that the number of low-mass $10^{6}<M_{\text {halo }} / M_{\odot}<10^{8}$ halos that host luminous satellite galaxies today is typically suppressed by 50 percent, with values ranging up to 90 percent in regions where the initial supersonic velocity is high. We show that this previouslyignored cosmological effect resolves most of the tension between the observed and predicted number of low-mass satellites in the Milky Way, obviating the need for any other mass-dependent star-formation suppression before reionization.
\end{abstract}

Subject headings: cosmology: theory — early universe — galaxies: formation — Galaxy: halo galaxies: statistics - Galaxy: structure

\section{INTRODUCTION}

A robust prediction of the Cold Dark Matter (CDM) cosmological paradigm of hierarchical clustering is that the halos of galaxies like the Milky Way should contain hundreds of satellite subhalos that could be expected to host observable galaxies (Kauffmann et al. 1993; Klypin et al. 1999; (Moore et al. 1999). The success of the $\Lambda \mathrm{CDM}$ model from the horizon-scale (Komatsu et al. 2011) to the small scale of the Lyman- $\alpha$ forest (Viel et al. 2008) suggests that this "missing satellites problem" is most likely the result of the details of galaxy-formation physics in low-mass halos rather than a manifestation of a deviation from the standard framework on small scales.

Potential solutions to the missing satellites problem have so far come in two flavors. One approach aims to reduce the intrinsic small-scale power in the CDM framework by positing alternatives to standard inflationary mechanisms for producing the initial perturbation spectrum (Kamionkowski \& Liddle 2000). Similarly, warm dark matter models naturally suppress the number of small, bound structures in the Universe (Polisensky \& Ricotti 2011). The alternative to modifying the cosmological framework is to invoke astrophysical explanations for suppressing star formation in low-mass galaxies. In particular, suppression of star formation by photo-heating by the ultraviolet (UV) background after reionization naturally leads to a starformation-efficiency cut-off at approximately the correct mass scale (Bullock et al. 2000; Somerville 2002). This can explain the number counts of "classical dwarf spheroidals" (dSphs) around the Milky Way $\left(M_{V} \lesssim-6\right.$; Koposov et al. 2009).

The Sloan Digital Sky Survey (SDSS; York et al. 2000)

1 Hubble fellow discovered many more smaller dSphs in the Milky Way's halo (e.g., Willman et al. 2005; Belokurov et al. 2006; Zucker et al. 2006; Belokurov et al. 2007; Irwin et al. 2007; Walsh et al. 2007). To explain the number counts of these new discoveries within $\Lambda \mathrm{CDM}$, it is necessary to invoke additional suppression of star formation in the lowest mass halos before reionization (e.g., Koposov et al. 2009), as star formation in these halos mostly ends at reionization due to photoionization. Specifically, the stellar mass in low-mass halos $\left(M \lesssim 10^{8} M_{\odot}\right)$ can only be $\lesssim 10^{-4}$ times the halo mass (Madau et al. 2008), an order of magnitude less than the stellar-mass fraction in higher mass halos. This is only $\approx 0.05 \%$ of the universal baryon fraction, while typically we would expect star formation efficiencies of at least a few percent, even when star formation only proceeds through $\mathrm{H}_{2}$ cooling (Bovill \& Ricotti 2009; Salvadori \& Ferrara 2009) and it is unclear whether radiative feedback from the first generation of stars suppresses pre-reionization star formation in low-mass halos or not (Haiman et al. 1996; Omukai \& Nishi 1999; Haiman et al. 2000; Ricotti et al. 2002a, b; Whalen et al. 2008).

In this paper we determine the influence of the previously-ignored effect of the relative velocity between baryons and dark matter at recombination on the stellar content of the smallest-mass galaxies. As was recently pointed out by Tseliakhovich \& Hirata (2010), the typical baryon-CDM relative velocity of $30 \mathrm{~km} \mathrm{~s}^{-1}$ at recombination is supersonic as the baryon sound speed at kinetic decoupling drops to $5 \mathrm{~km} \mathrm{~s}^{-1}$. While the relative velocity decays as $\propto a^{-1}$, where $a$ is the scale factor, the supersonic baryon flow has a profound effect on the formation of the first structures (Tseliakhovich et al. 2011; Stacv et al. 2011; Maio et al. 2011; Greif et al. 2011; Naoz et al. 2012; Visbal et al. 
2012; O'Leary \& McQuinn 2012), which through the effects of hierarchical clustering might persist today (e.g., in the baryon acoustic feature, Dalal et al. 2010), and it could also give rise to $\mathrm{CMB} B$-modes if the effect persists during the epoch of reionization (Ferraro et al. 2012). We show that the supersonic baryon flow has a large effect on both the number and the baryon content of halos with masses between the $\mathrm{H}_{2}$ cooling limit $(\approx$ $10^{6} M_{\odot}$; Tegmark et al. 1997) and the photo-ionization scale $\left(\approx 10^{7} M_{\odot}\right.$ at $z=11$, Gnedin 2000). This typically reduces the number of satellites by 50 up to 90 percent at $M_{V} \approx-1$.

The outline of this paper is as follows. In $\oint 2$ we review the supersonic baryon-CDM relative velocity effect. We then compute the pre-reionization mass and luminosity functions in $\S$. We discuss the low-redshift behavior of the satellite luminosity function in $\S$ 4. Our conclusions are in $\S$. We assume cosmological parameters matching the WMAP 7-year data (Komatsu et al. 2011): $\Omega_{b, 0}=$ $0.0456, \Omega_{c, 0}=0.227, z_{\text {eq }}=3232, H_{0}=70.4 \mathrm{~km} \mathrm{~s}^{-1}$ $\mathrm{Mpc}^{-1}, \Delta^{2} \zeta\left(k=0.002 \mathrm{Mpc}^{-1}\right)=2.44 \times 10^{-9}$, and $n_{s}=$ 0.963 .

\section{LINEAR EVOLUTION OF DENSITY PERTURBATIONS IN THE PRESENCE OF A SUPERSONIC FLOW}

After kinetic decoupling $(z \approx 1020$; Eisenstein \& Hu 1998) the baryonic sound speed drops to $5 \mathrm{~km} \mathrm{~s}^{-1}$, while the baryons move relative to the CDM with a typical velocity of $30 \mathrm{~km} \mathrm{~s}^{-1}$. As originally pointed out by Tseliakhovich \& Hirata (2010), this means that secondorder terms such as $\mathbf{v} \cdot \nabla \delta$ and $\mathbf{v} \cdot \nabla \mathbf{v}$, which are typically neglected to first order, become as large as firstorder terms in the continuity and Navier-Stokes equations that describe the evolution of inhomogeneities in the baryons and CDM after recombination. We follow the treatment of Tseliakhovich et al. (2011), who shows that the supersonic relative flow $\mathbf{v}_{b c}$ is homogeneous over a few comoving Mpc, with $\mathbf{v}_{b c}$ drawn from a Gaussian with a variance per axis of $\sigma_{b c}^{2} / 3$ with $\sigma_{b c}=30 \mathrm{~km} \mathrm{~s}^{-1}$ at kinetic decoupling. The evolution equations for the relative CDM $\left(\delta_{c}\right)$ and the baryon $\left(\delta_{b}\right)$ perturbations can be written in the baryon rest frame in a $\sim$ comoving Mpc patch with a supersonic flow $\mathbf{v}_{b c}$ as

$$
\begin{aligned}
\frac{\partial \delta_{c}}{\partial t}= & \frac{i}{a} \mathbf{v}_{b c} \cdot \mathbf{k} \delta_{c}-\theta_{c} \\
\frac{\partial \theta_{c}}{\partial t}= & \frac{i}{a} \mathbf{v}_{b c} \cdot \mathbf{k} \theta_{c}-\frac{3 H_{0}^{2}}{2} \frac{\Omega_{m, 0}}{a^{3}}\left(\frac{\Omega_{c, 0}}{\Omega_{m, 0}} \delta_{c}+\frac{\Omega_{b, 0}}{\Omega_{m, 0}} \delta_{b}\right) \\
& \quad-2 H \theta_{c} \\
\frac{\partial \delta_{b}}{\partial t}=- & \theta_{b} \\
\frac{\partial \theta_{b}}{\partial t}=-\frac{3 H_{0}^{2}}{2} \frac{\Omega_{m, 0}}{a^{3}}\left(\frac{\Omega_{c, 0}}{\Omega_{m, 0}} \delta_{c}+\frac{\Omega_{b, 0}}{\Omega_{m, 0}} \delta_{b}\right) & \quad-2 H \theta_{b}+\frac{k^{2}}{a^{2}} \frac{k_{B} T_{b}}{\mu m_{H}}\left(\delta_{b}+\delta_{T_{b}}\right),
\end{aligned}
$$

where we write the baryonic sound speed as $c_{s}^{2}=$ $k_{B} T_{b}\left(\delta_{b}+\delta_{T_{b}}\right) / \mu m_{H} \delta_{b}, \quad \theta$ is the velocity divergence, $\Omega_{m, 0}=\Omega_{b, 0}+\Omega_{c, 0}, \mu=1.22$ is the mean molecular weight including a helium mass fraction of $0.24, m_{H}$ is the mass of the hydrogen atom, and $T_{b}$ and $\delta_{T_{b}}$ are the mean baryon temperature and its relative fluctuation, respectively. The evolution of $T_{b}$ is given by (Tseliakhovich \& Hirata 2010)

$$
T_{b}=\frac{T_{\mathrm{CMB}, 0}}{a}\left[1+\frac{a /(1 / 119)}{1+(1 / 115 / a)^{3 / 2}}\right]^{-1},
$$

where $T_{\mathrm{CMB}, 0}=2.726 \mathrm{~K}$. The temperature fluctuation evolves according to Barkana \& Loeb 2005; Naoz \& Barkana 2005)

$$
\frac{\partial \delta_{T_{b}}}{\partial t}=-\frac{2}{3} \theta_{b}-\frac{x_{e}(t)}{t_{\gamma}} \frac{1}{a^{4}} \frac{T_{\gamma}}{T_{b}} \delta_{T_{b}}
$$

where $x_{e}(t)$ is the free electron fraction out of the total number density of gas particles, $t_{\gamma}=8.55 \times 10^{-13}$ $\mathrm{yr}^{-1}, T_{\gamma}$ is the mean photon temperature, and we have neglected the photon inhomogeneities compared to Naoz \& Barkana (2005). We obtain the free electron fraction $x_{e}(t)$ using RECFAST (Seager et al. 1999, $2000)$.

We solve the complex system of Equations (13) by taking initial conditions for the matter inhomogeneities and velocities from CAMB2 (Lewis et al. 2000) at kinetic decoupling $(z=1020)$ and setting up the initial inhomogeneities in the baryon temperature following the approximation done in Naoz \& Barkana (2005) by requiring $\partial \delta_{T_{b}} / \partial t=\partial \delta_{T_{\gamma}} / \partial t$, where $\delta_{T_{\gamma}}$ is the relative photon temperature perturbation. This approximation is justified because of tight thermal coupling between the baryons and the photons and it has been shown to affect the power spectra only by a fraction of a percent at $z=1020$. The relative velocity in Equation (1) decays as $\mathbf{v}_{b c} \propto a^{-1}$.

\section{LUMINOSITY FUNCTION BEFORE REIONIZATION}

We follow the procedure of Tseliakhovich et al. (2011) to calculate the halo mass function and the baryonic content of low-mass halos before reionization in the presence of a supersonic baryon-CDM flow. We calculate the halo mass function using the Extended Press-Schechter formalism (Bond et al. 1991; Bower 1991; Lacev \& Cole 1993)

$$
\frac{\mathrm{d} N}{\mathrm{~d} M_{\text {halo }}}\left(M_{\text {halo }} ; v_{b c}\right)=\frac{\bar{\rho}_{0}}{M_{\text {halo }}}\left|\frac{\mathrm{d} S}{\mathrm{~d} M_{\text {halo }}}\right| f\left(\delta_{c}(z), S\right) \text {, }
$$

where $\bar{\rho}_{0}$ is the mean matter density in the universe and $S$ is the usual variance given by

$$
S\left(M ; v_{b c}\right)=\int d \ln k \Delta_{m}^{2}\left(k ; v_{b c}\right)|W(k ; R)|^{2}
$$

Here $\Delta_{m}^{2}\left(k ; v_{b c}\right)$ is the matter power spectrum computed using Equations (13). The transfer functions computed using Equations (1) 3) depend on the angle between $\mathbf{k}$ and $\mathbf{v}_{b c}$. In what follows, we average all solutions for $\left|\delta_{b}\right|$ and $\left|\delta_{c}\right|$ over this angle and give results for $v_{b c}=\left|\mathbf{v}_{b c}\right| . W(k ; R)$ is the tophat window function and the initial comoving radius $R$ corresponds to a halo

\footnotetext{
2 http://camb.info/.
} 


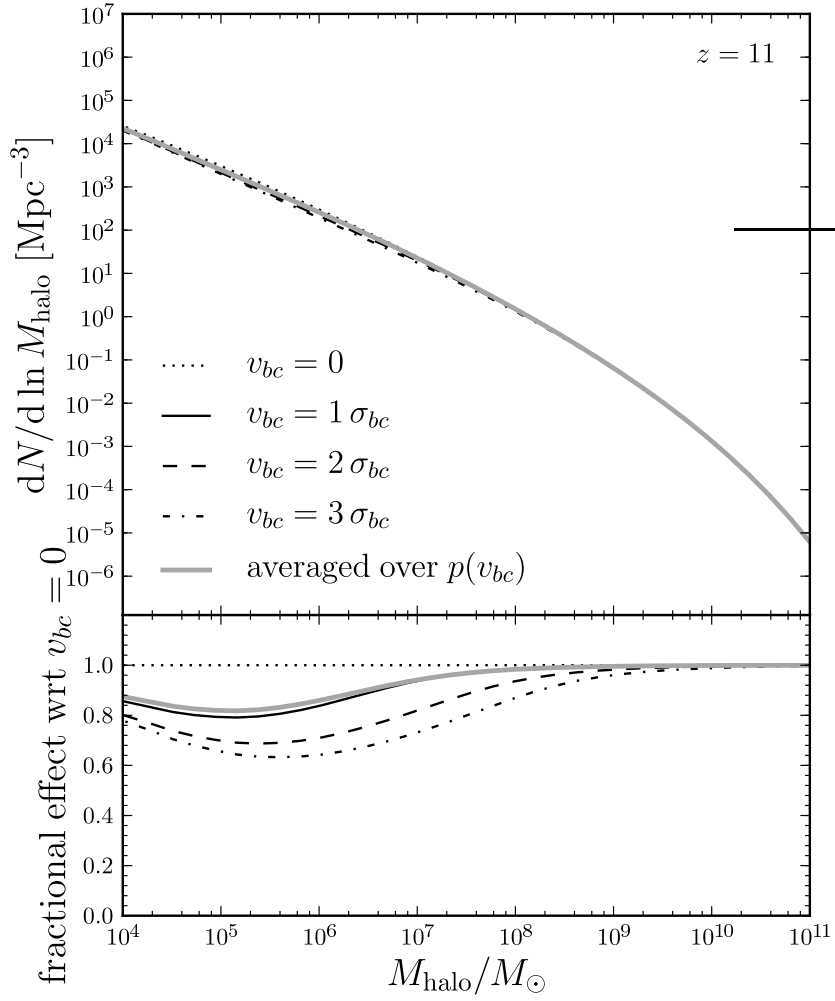

Fig. 1.- Halo mass function at $z=11$ with a relative baryonCDM velocity of $v_{b c}=0$ and $v_{b c} \neq 0.1 \sigma_{b c}$ corresponds to approximately $30 \mathrm{~km} \mathrm{~s}^{-1}$ at decoupling $(z=1020)$. The globally-averaged effect obtained by averaging over the Gaussian probability distribution function of $\mathbf{v}_{b c}$ is shown as the gray curve.

of mass $M_{\text {halo }}=4 \pi R^{3} \bar{\rho}_{0} / 3$. We use the functional form from Sheth \& Tormen (1999) for $f\left(\delta_{c}(z), S\right)$ :

$$
f\left(\delta_{c}(z), S\right)=A \frac{\nu}{S} \sqrt{\frac{a}{2 \pi}}\left[1+\frac{1}{\left(a \nu^{2}\right)^{q}}\right] e^{-a \nu^{2} / 2},
$$

where $\nu=\delta_{c}(z) / \sqrt{S}, a=0.75, q=0.3, A=0.322$ (Sheth \& Tormen 2002), and $\delta_{c}(z)=1.67$ is the critical density of collapse at $z \approx 10$.

Constraints from the Cosmic Microwave Background on the epoch of reionization are consistent with a redshift of reionization of $z=11$ (Komatsu et al. 2011; Zahn et al. 2011) (defined as the redshift at which reionization would begin if the universe was reionized instantaneously). The halo mass function at $z=11$ calculated using the procedure given above is shown in FIG.11 This figure shows that the effect of the supersonic baryon-CDM velocity typically suppresses the number of dark matter halos by 10 to 20 percent in the range $10^{5}<M_{\text {halo }} / M_{\odot}<10^{7}$ at $z=11$. $N$-body simulations by Naoz et al. (2012) show a similar level of suppression in the mass function at $z=11$.

To estimate the stellar mass in low-mass subhalos at reionization, we use the results from Naoz \& Barkana (2007) and Naoz et al. (2009). These studies have shown that the gas fraction in halos at high-redshift is suppressed with respect to the universal baryon fraction by the combined effect of the remaining suppression after recombination in the baryon density perturbations on small scales (Naoz \& Barkana 2007; Barkana \& Loeb 2011) and the baryonic pressure, which gives rise to a

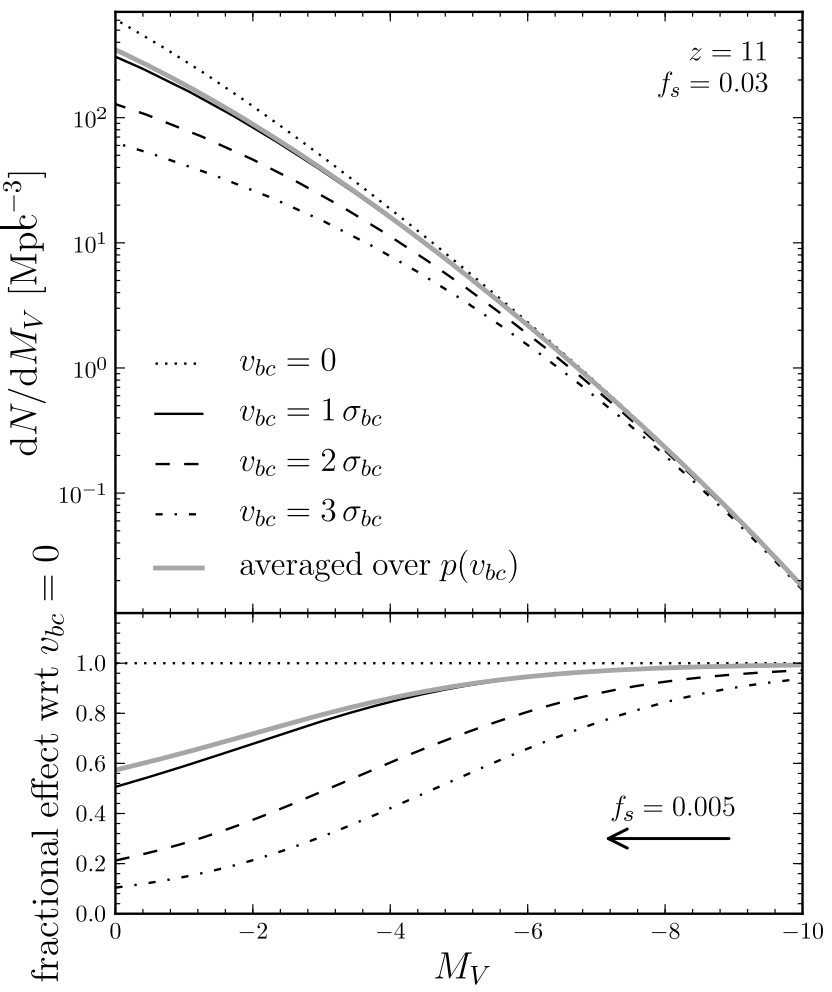

FIG. 2.- Luminosity function at $z=11$ using the luminosity that the halos would have today. This is the total effect on the luminosity function from the combination of the suppression of the halo mass function and the reduction in the gas fraction due to $v_{b c} \neq 0$. The average case is shown in the gray curve. The separate contributions to the suppression for $v_{b c} \neq 0$ from the halo mass function and gas fraction are shown in FIG. 3 The arrow in the bottom panel shows how far each curve moves when lowering the star-formation efficiency to $f_{s}=0.005$.

redshift-dependent 'filtering' scale (Gnedin \& Hui 1998; Gnedin 2000; Naoz \& Barkana 2007). This filtering scale corresponds to the length scales below which the baryonto-total-matter fluctuation drops substantially below its large-scale value, and simulations find that it characterizes the minimum halo mass that can retain its gas (Naoz et al. 2009; O'Leary \& McQuinn 2012). To determine the filtering scale $k_{F}$ as a function of the supersonic flow velocity $\mathbf{v}_{b c}$ we fit the functional form

$$
\frac{\left|\delta_{b}\right|}{\left|\delta_{\mathrm{tot}}\right|}=\left(1+r_{\mathrm{LSS}}\right)\left(1+\frac{1}{n} \frac{\left(k^{2} / k_{F}^{2}\right)}{1+r_{\mathrm{LSS}}}\right)^{-n}
$$

where $\left|\delta_{\text {tot }}\right|=\left(\Omega_{b, 0}\left|\delta_{b}\right|+\Omega_{c, 0}\left|\delta_{c}\right|\right) / \Omega_{m, 0}, r_{\text {LSS }}$ is determined from the behavior of $\left|\delta_{b}\right| /\left|\delta_{\text {tot }}\right|$ on larger scales, $1 \leq k \mathrm{Mpc} \leq 10$, and $k_{F}$ and $n$ are subsequently fit at $k \geq 1 \mathrm{Mpc}^{-1}$.

Using the filtering scale $k_{F}$, the filtering mass is defined as (Naoz \& Barkana 2007)

$$
M_{F}\left(M_{\text {halo }} ; v_{b c}\right)=\frac{4 \pi}{3} \bar{\rho}_{0}\left(\frac{\pi}{k_{F}}\right)^{3}
$$

Using the filtering mass, we can estimate the gas fraction at redshifts $z \geq 11$ as (Naoz et al. 2009)

$$
f_{\text {gas }}\left(M ; v_{b c}\right)=f_{b, 0}\left[1+\left(2^{\alpha / 3}-1\right)\left(\frac{M_{F}}{M}\right)^{\alpha}\right]^{-3 / \alpha}
$$




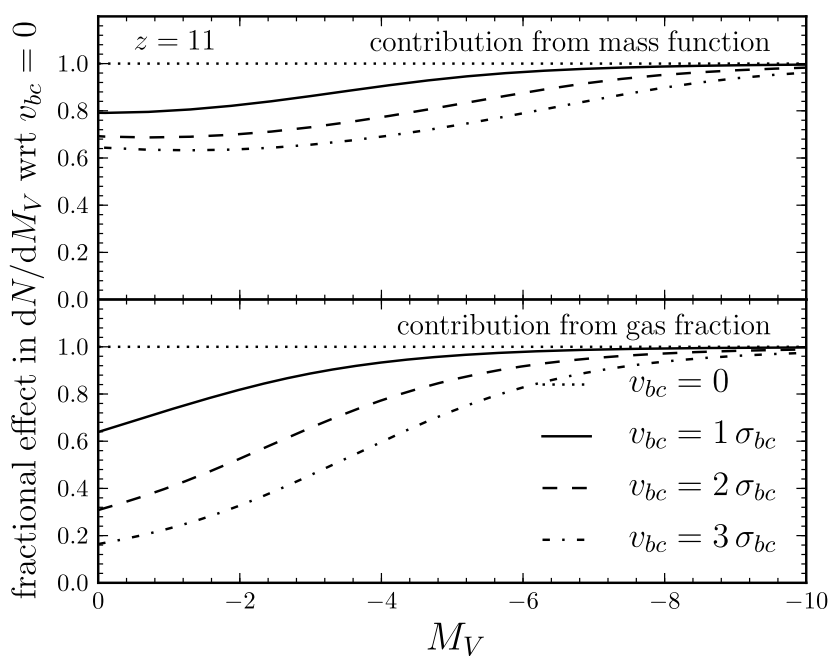

FIG. 3.- Separate contributions to the difference in the $z=11$ luminosity function in FIG. 2 between $v_{b c}=0$ and $v_{b c} \neq 0$ from the suppression in the halo mass function in FIG. 1 and the suppression in the gas fraction.

where $f_{b, 0}$ is the gas fraction in the high-mass limit. $f_{b, 0}$ is typically lower than the universal baryon fraction because density perturbations in the baryons at high redshift remain suppressed due to the lingering effect of the coupling between baryons and photons before recombination. In Barkana \& Loeb (2011), the authors show that $f_{b, 0} \approx\left(1+3.2 r_{\mathrm{LSS}}\right) \Omega_{b, 0} / \Omega_{m, 0}$. Following Tseliakhovich et al. (2011), we use $\alpha=0.7$, even though this value of $\alpha$ was calculated for halos at $z \approx 20$. As our main objective is to show the difference between the $v_{b c}=0$ and $v_{b c} \neq 0$ cases, the exact functional form in Equation (9) does not matter greatly.

We assume that a fraction $f_{s}$ of the gas in a haloindependent of halo mass - turns into stars, such that the stellar mass is given by

$$
M_{s}\left(M_{\text {halo }} ; v_{b c}\right)=f_{s} f_{\text {gas }}\left(M_{\text {halo }} ; v_{b c}\right) M_{\text {halo }} .
$$

We convert this stellar mass into a luminosity by assuming that this stellar mass is turned into stars with very low metallicity $Z=Z_{\odot} / 200$. Today, such a population would shine with an absolute magnitude $M_{V}=6.7$ per solar mass (Madau et al. 2008; Bruzual \& Charlot 2003) and it is this luminosity that we use to show the resulting luminosity function.

FIG. 2] shows the luminosity function at $z=11$ using the luminosity that the halos would have today. That is, it is the luminosity function we would observe today if the low-mass halos were frozen in their pre-reionization state and there was no evolution in the number of halos. We will study their evolution in $\S 4$

FIG. 2 assumes a star formation efficiency of $f_{s}=0.03$, which gives a total-to-stellar mass at the high-mass end of $10^{-2.8}$. The arrow in the bottom panel of FIG. 2 shows the effect of lowering the star-formation efficiency to $f_{s}=0.005$. In this case, halos of a given luminosity come from higher total-mass halos, where the effect of the supersonic baryon-CDM velocity is smaller, thus shifting the effect toward smaller luminosities.

FIG. 3. shows the relative contribution to the total effect in FIG. 2 from the suppression of the mass function in the presence of the supersonic baryon-CDM flow (see
FIG. 1) and the suppression of the baryon fractions. It is interesting to note that the suppression in the accretion of baryons onto dark matter halos is the main driver of the suppression of low-luminosity halos.

McQuinn \& O'Leary (2012) find that shock heating can raise the baryon temperature by approximately 10 percent compared to the evolution that we assume. If we approximate the evolution in $T_{b}$ that they find by raising $T_{b}$ by 10 percent at $z<20$, we find a negligible influence on the matter power spectrum and filtering mass at $z=11$, such that the conclusions of this section are unaffected by shock heating.

\section{PRESENT-DAY SATELLITE LUMINOSITY FUNCTION}

In this section we quantify how the suppression at the faint end of the luminosity function before reionization affects the present-day subhalo luminosity function for a Milky-Way sized halo. We estimate the satellite luminosity function by running merger-tree simulations using the Extended Press-Schechter formalism of Lacey \& Cole (1993) and Cole et al. (2000) for a parent halo of mass $M=10^{12} M_{\odot}$ (Xue et al. 2008). We create 100 merger trees for each of $v_{b c}=0,1,2$, and $3 \sigma_{b c}$ by computing the $z=0 S\left(M ; v_{b c}\right)$ as before and using the linear overdensity for collapse $\delta_{c}=1.67$, extrapolated to $z=0$ using the growth factor (Eke et al. 1996; Carroll et al. 1992). We resolve subhalos down to a resolution mass of $M_{\text {res }}=10^{6} M_{\odot}$ and run the simulation until $z=11$. As we are only interested in low-mass subhalos, we only track the mass evolution of subhalos, without resolving it into sub-subhalos. For each subhalo, we record the pre-reionization mass $M(z=11)$, defined as the mass of the subhalo at $z=11$, and the mass at the time of the merger with the parent halo $M\left(z=z_{\text {acc }}\right)$.

We do not follow the dynamical and mass evolution of the subhalos after they merge with the parent halo. This approximation assumes that low-mass $\left(<10^{8} M_{\odot}\right)$ satellites do not get tidally disrupted and do not lose a significant amount of stellar mass after merging with the parent halo. High-resolution $N$-body simulations find that only a few percent of $M>10^{7} M_{\odot}$ subhalos are tidally destroyed between $z=1$ and $z=0$ and that subhalos with $M<10^{8} M_{\odot}$ retain most of their total mass (Diemand et al. 2007). Even when the outer parts of the subhalos are tidally stripped, the stars and the inner part of the subhalo are stripped only at the last stage of the tidal disruption of the subhalo (Peñarrubia et al. 2008), such that most of the stellar mass is retained, even if a significant part of the dark matter halo is tidally stripped. Therefore, to a good approximation, the stellar-mass function of low-mass satellites should not be strongly affected by the effects of tidal stripping and disruption.

We compute the luminosity of each subhalo by using the prescription of Equation (10) applied to the pre-reionization mass $M(z=11)$ to calculate the prereionization stellar-mass and turning this into a luminosity again using $M_{V}=6.7$ per solar mass. We calculate the post-reionization stellar mass for each subhalo by using a star-formation efficiency that takes into account suppression by photoionization (Gnedin 2000; Hoeft et al. 2006; Okamoto et al. 2008) and that assumes that the effect of the supersonic baryon-CDM velocity has no influence on star formation after reionization: 


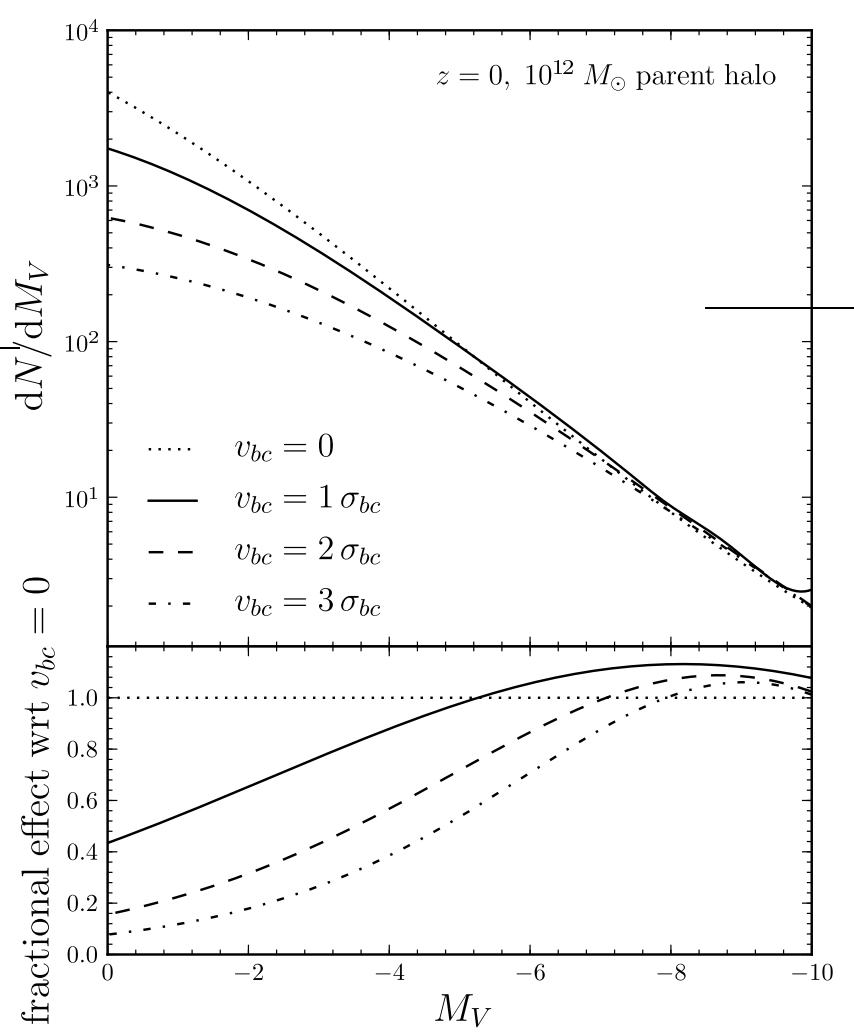

FIG. 4.- Satellite luminosity function at $z=0$ for a Milky-Way size halo $\left(M=10^{12} M_{\odot}\right)$. The curves show the average luminosity function of 100 merger trees for each value of $v_{b c}$. The bottom panel shows the fractional effect with respect to $v_{b c}=0$.

$$
M_{s}(z<11)=f_{*} \frac{\left(M\left(z=z_{\mathrm{acc}}\right)-M(z=11)\right)}{\left(1+0.26\left(V_{\text {crit }} / V_{\text {circ }}\left(z_{\text {acc }}\right)\right)^{3}\right)^{3}},
$$

where $f_{*}=10^{-3} / 6.25$ and $V_{\text {crit }}=35 \mathrm{~km} \mathrm{~s}^{-1}$ (as in Koposov et al. 2009). We calculate $V_{\text {circ }}\left(z_{\text {acc }}\right)$ by using the virial radius of Equation (1) in Koposov et al. (2009). The post-reionization contribution to the total stellar luminosity of a satellite is then calculated by assuming a solar mass-to-light ratio (Martin et al. 2008).

The luminosity function of satellites of a Milky-Way type halo computed in this way is shown in FIG. 4. A comparison between this figure and FIG. 2 confirms that the pre-reionization effect on the suppression of the luminosity function at $z=11$ translates into almost the same suppression in the satellite luminosity function at $z=0$. Additional suppression of the luminosity function results from the fact that in a region of a highly supersonic baryon-CDM flow, structure formation is delayed, such that a larger fraction of the present-day mass of a halo was accreted after the epoch of reionization, where it is affected by the suppressed gas accretion and cooling in the presence of the photoionizing background after reionization. This additional effect also explains the slight increase in the number of brighter satellites in FIG. 4. At the bright end - where pre-reionization suppression due to the supersonic baryon-CDM flow is small - a larger fraction of a satellite's mass is accreted after reioinization where we have assumed a mass-to-light ratio appro-

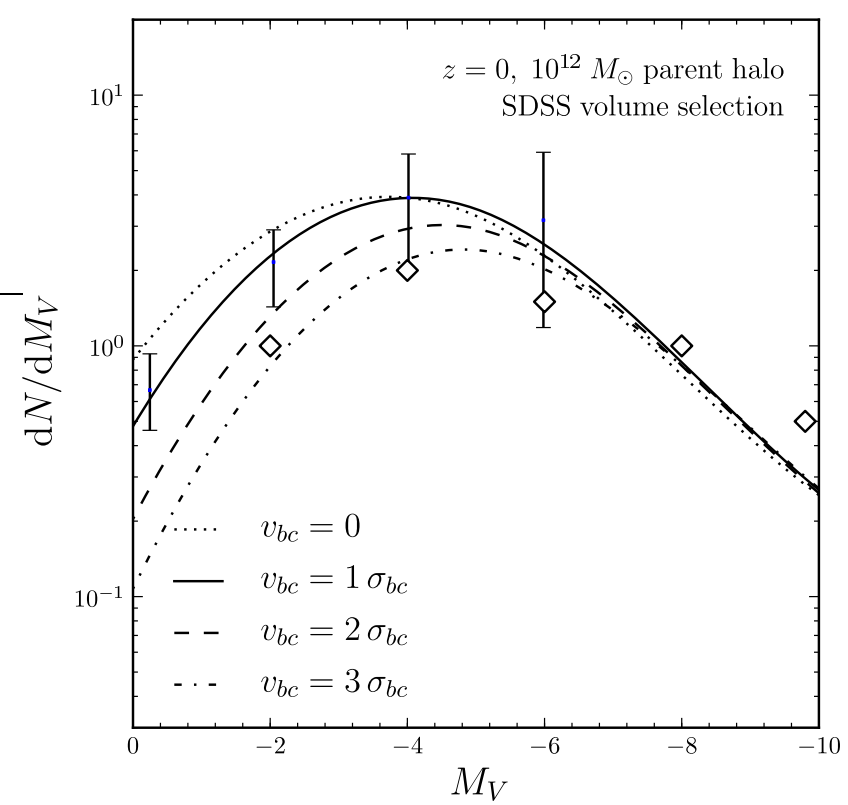

FIG. 5.- Luminosity function of satellite galaxies of the Milky Way observable by the SDSS. The diamond data points are taken from Koposov et al. (2009). The error bars on the $v_{b c}=1 \sigma_{b c}$ model curve show the 68 percent spread in the merger trees at luminosities where the predictions with different $v_{b c}$ differ; this spread is similar for all four model curves. The error bars are highly correlated; for example, the correlation between the $M_{V}=-4$ and $M_{V}=-2$ error bars is 0.75 . For this figure we assume $f_{\mathrm{s}}=0.01$.

priate for a metal-poor population rather than that of an extremely metal-poor population, which we assumed for pre-reionization star formation. This discontinuity, which is an artifact of the simplicity of our assumptions, stretches the $v_{b c} \neq 0$ curves toward the brighter end, leading to an increased number of bright satellites.

To compare the predicted satellite luminosity function to the observed luminosity function of satellites of the Milky Way (e.g., Koposov et al. 2008), we assume that the spatial distribution of the satellites follows a NavarroFrenk-White profile with a scale radius of $30 \mathrm{kpc}$; in accordance with numerical simulations (Diemand et al. 2007), we anti-bias this distribution radially by multiplying by the Galactocentric radius. We then calculate the observational fraction by using the simple model for the SDSS satellite selection function of Koposov et al. (2009), where a satellite of absolute magnitude $M_{V}$ can be detected out to a distance of $D_{\max }=10^{1.1-0.228 M_{V}}$ $\mathrm{kpc}$ from the Sun (assumed to be $8 \mathrm{kpc}$ from the Galactic center, Bovy et al. 2009), integrating out to a virial radius of $260 \mathrm{kpc}$. We multiply this selection fraction by 0.194 to account for the partial sky coverage of the SDSS. All satellites down to $M_{V} \approx-6$ could be observed throughout the virial volume by the SDSS.

The luminosity function of satellites detectable by the SDSS thus computed is shown in FIG. 5. Even though, for this figure we have assumed a star-formation efficiency $f_{\mathrm{s}}$ of only 0.01 , it is clear that the supersonic baryon-CDM velocity has a large effect on the faint end of this luminosity function that significantly lowers the predicted fraction of satellites at $M_{V}>-6$. Thus, the inefficient gas accretion at high redshift induced by a large supersonic flow has a large and lasting effect on the abundance of luminous satellites for a galaxy like the 
Milky Way.

We do not show the upper limit of $\mathrm{d} N / \mathrm{d} M_{V}<0.1$ at $M_{V}=0$ from Koposov et al. (2009) in FIG. 5 as the predictions at $M_{V}=0$ strongly depend on the exact form of the SDSS selection function. At $M_{V}=0$, the maximum distance out to which a satellite can be detected by the SDSS is approximately $12 \mathrm{kpc}$. As we do not follow the dynamical evolution of the satellites in the parent halo, our simulations do not capture the fact that those satellites that come within $20 \mathrm{kpc}$ of the Galactic center have a much larger chance of being tidally disrupted, such that our predictions at $M_{V}=0$ over-estimate the number of observable satellites.

\section{CONCLUSION}

As pointed out by Tseliakhovich \& Hirata (2010), at recombination, baryons move with a typical supersonic velocity of $30 \mathrm{~km} \mathrm{~s}^{-1}$ with respect to the dark matter. While this relative baryon-CDM velocity decays as $\propto a^{-1}$, it has a large effect on the formation of the first structures in the Universe. We have investigated the effect on the present-day abundance of luminous low-mass satellite galaxies $\left(\lesssim 10^{8} M_{\odot}\right)$ of a Milky-Way size galaxy. One would expect the supersonic baryon-CDM velocity to affect the abundance and luminosity of low-mass satellites, as these satellites must form many of their stars before reionization because photo-heating by the UV background after reionization suppresses star formation in halos with circular velocities $\lesssim 30 \mathrm{~km} \mathrm{~s}^{-1}$. The effect of the supersonic baryon-CDM flow should be a standard cosmological effect that needs to be taken into account when predicting the number of luminous satellite galaxies - the only unknown being the initial value of the relative velocity in the patch of the Universe that is being considered. Additionally, we are motivated by the apparent discrepancy between the observed number of dwarf satellite galaxies in the Milky Way at $M_{V} \gtrsim-4$, which indicates that to fully explain the missing satellites problem, star formation before reionization needs to be suppressed, in addition to the - now standardsuppression by photo-heating after reionization.

We have shown by following the linear evolution of density fluctuations while taking into account the nonlinear effect of the supersonic baryon-CDM flow, that the stellar-mass function before reionization $(z \approx 11)$ is significantly suppressed at the low-luminosity end, with a typical suppression by 50 percent for luminosities today of $M_{V} \gtrsim-4$, going up to 90 percent for regions with a high initial supersonic relative velocity. This suppression is mainly the result of the reduced accretion of gas onto low-mass halos due to the supersonic flow, with a small contribution from the overall suppression of the halo mass function at $10^{5}<M_{\text {halo }} / M_{\odot}<10^{7}$. While these calculations use the linear evolution equations for perturbations, cosmological high-resolution hydrodynami- cal simulations of the formation of the first structures have shown that the approximations of $\S \$ 2$ and 3 adequately describe the formation and baryon fraction of the first galaxies (Naoz et al. 2009; O'Leary \& McQuinn 2012), potentially even underestimating the suppression in the star-forming gas fraction at the high-mass end ( $\approx 10^{7} M_{\odot} ;$ McQuinn \& O'Leary 2012).

To determine whether the effect of the supersonic baryon-CDM flow on the abundance of luminous lowmass galaxies persists to the satellite luminosity function of a Milky-Way sized halo today, we have run extended Press-Schechter simulations of the merger history of a $10^{12} M_{\odot}$ halo, taking into account the effect of the supersonic flow on the halo mass function and star formation prior to reionization. We found that the effect largely remains the same and that the number of satellite galaxies with $M_{V} \gtrsim-4$ in a Milky-Way sized halo is typically suppressed by 50 percent. When then predicting the observed number of faint satellites of the Milky Way that could have been observed by the SDSS - multiplying the predicted counts by the fraction of the effective volume of the Milky Way observed by the SDSS - we find that a typical initial relative velocity of $\approx 30 \mathrm{~km} \mathrm{~s}^{-1}$ alleviates most of the discrepancy at the faint end $\left(M_{V}>-6\right)$ between the observed number of satellites and that predicted by a model without pre-reionization suppression of star formation, without applying any additional massdependent reduction in star-formation efficiency, e.g., due to the radiative feedback from the first stars. Therefore, the effect of the supersonic baryon-CDM flow naturally provides the amount and mass-dependence of the pre-reionization suppression of star-formation efficiency needed to explain the observed luminosity function of Milky-Way satellites. The limited detection efficiency of the SDSS for low-luminosity galaxies is such that satellites at $M_{V}=-2$ can only be detected out to approximately $40 \mathrm{kpc}$, such that a large fraction of the virial volume of the Milky Way remains unexplored at these low-luminosities. Next-generation surveys such as the LSST will be able to observe $M_{V}=0$ satellite galaxies out the virial radius of the Milky Way (Tollerud et al. 2008), and the effect of the supersonic baryon-CDM flow should lead to a clear suppression in the number of lowluminosity satellites.

Acknowledgments: It is a pleasure to thank Simone Ferraro, Wayne $\mathrm{Hu}$, and Matias Zaldarriaga for useful comments. Support for Program number HSTHF-51285.01-A was provided by NASA through a Hubble Fellowship grant from the Space Telescope Science Institute, which is operated by the Association of Universities for Research in Astronomy, Incorporated, under NASA contract NAS5-26555. CD was supported by the National Science Foundation (NSF) Grant No. AST0807444, NSF Grant No. PHY-0855425, and the Raymond and Beverly Sackler Funds.

\section{REFERENCES}

Barkana, R., \& Loeb, A. 2005, MNRAS, 363, L36

-. 2011, MNRAS, 415, 3113

Belokurov, V., et al. 2006, ApJ, 647, L111

-. 2007, ApJ, 654, 897

Bond, J. R., Cole, S., Efstathiou, G., \& Kaiser, N. 1991, Astrophys. J., 379, 440

Bovill, M. S., \& Ricotti, M. 2009, ApJ, 693, 1859
Bovy, J., Hogg, D. W., \& Rix, H.-W. 2009, ApJ, 704, 1704

Bower, R. G. 1991, Mon. Not. Roy. Astron. Soc., 248, 332

Bruzual, G., \& Charlot, S. 2003, MNRAS, 344, 1000

Bullock, J. S., Kravtsov, A. V., \& Weinberg, D. H. 2000, ApJ, 539,517

Carroll, S. M., Press, W. H., \& Turner, E. L. 1992, ARA\&A, 30, 499 
Cole, S., Lacey, C. G., Baugh, C. M., \& Frenk, C. S. 2000, MNRAS, 319, 168

Dalal, N., Pen, U.-L., \& Seljak, U. 2010, J. Cosmol. Astropart. Phys., 11, 7

Diemand, J., Kuhlen, M., \& Madau, P. 2007, ApJ, 667, 859

Eisenstein, D. J., \& Hu, W. 1998, ApJ, 496, 605

Eke, V. R., Cole, S., \& Frenk, C. S. 1996, MNRAS, 282, 263

Ferraro, S., Smith, K. M., \& Dvorkin, C. 2012, Phys.Rev., D85, 043523

Gnedin, N. Y. 2000, ApJ, 542, 535

Gnedin, N. Y., \& Hui, L. 1998, MNRAS, 296, 44

Greif, T. H., White, S. D. M., Klessen, R. S., \& Springel, V. 2011, ApJ, 736, 147

Haiman, Z., Abel, T., \& Rees, M. J. 2000, ApJ, 534, 11

Haiman, Z., Rees, M. J., \& Loeb, A. 1996, ApJ, 467, 522

Hoeft, M., Yepes, G., Gottlöber, S., \& Springel, V. 2006, MNRAS, 371, 401

Irwin, M. J., et al. 2007, ApJ, 656, L13

Kamionkowski, M., \& Liddle, A. R. 2000, Phys. Rev. Lett., 84, 4525

Kauffmann, G., White, S. D. M., \& Guiderdoni, B. 1993, MNRAS, 264, 201

Klypin, A., Kravtsov, A. V., Valenzuela, O., \& Prada, F. 1999, ApJ, 522, 82

Komatsu, E., et al. 2011, ApJS, 192, 18

Koposov, S., et al. 2008, ApJ, 686, 279

Koposov, S. E., Yoo, J., Rix, H.-W., et al. 2009, ApJ, 696, 2179

Lacey, C., \& Cole, S. 1993, MNRAS, 262, 627

Lewis, A., Challinor, A., \& Lasenby, A. 2000, ApJ, 538, 473

Madau, P., Kuhlen, M., Diemand, J., et al. 2008, ApJ, 689, L41

Maio, U., Koopmans, L. V. E., \& Ciardi, B. 2011, MNRAS, 412, L40

Martin, N. F., de Jong, J. T. A., \& Rix, H.-W. 2008, ApJ, 684, 1075

McQuinn, M., \& O'Leary, R. M. 2012, ApJ, submitted, arXiv: 1204.1345

Moore, B., Ghigna, S., Governato, F., et al. 1999, ApJ, 524, L19

Naoz, S., \& Barkana, R. 2005, MNRAS, 362, 1047

-. 2007, MNRAS, 377, 667
Naoz, S., Barkana, R., \& Mesinger, A. 2009, MNRAS, 399, 369

Naoz, S., Yoshida, N., \& Gnedin, N. Y. 2012, ApJ, 747, 128

Okamoto, T., Gao, L., \& Theuns, T. 2008, MNRAS, 390, 920

O'Leary, R. M., \& McQuinn, M. 2012, ApJ, submitted, arXiv: 1204.1344

Omukai, K., \& Nishi, R. 1999, ApJ, 518, 64

Peñarrubia, J., Navarro, J. F., \& McConnachie, A. W. 2008, ApJ, 673, 226

Polisensky, E., \& Ricotti, M. 2011, Phys.Rev., D83, 043506

Ricotti, M., Gnedin, N. Y., \& Shull, J. M. 2002a, ApJ, 575, 33

-. 2002b, ApJ, 575, 49

Salvadori, S., \& Ferrara, A. 2009, MNRAS, 395, L6

Seager, S., Sasselov, D. D., \& Scott, D. 1999, ApJ, 523, L1

—. 2000, ApJS, 128, 407

Sheth, R. K., \& Tormen, G. 1999, MNRAS, 308, 119

—. 2002, MNRAS, 329, 61

Somerville, R. S. 2002, ApJ, 572, L23

Stacy, A., Bromm, V., \& Loeb, A. 2011, ApJ, 730, L1

Tegmark, M., Silk, J., Rees, M. J., et al. 1997, ApJ, 474, 1

Tollerud, E. J., Bullock, J. S., Strigari, L. E., \& Willman, B. 2008, ApJ, 688, 277

Tseliakhovich, D., Barkana, R., \& Hirata, C. M. 2011, MNRAS, 418, 906

Tseliakhovich, D., \& Hirata, C. 2010, Phys. Rev. D, 82, 083520

Viel, M., Becker, G. D., Bolton, J. S., et al. 2008, Phys. Rev. Lett., 100, 041304

Visbal, E., Barkana, R., Fialkov, A., Tseliakhovich, D., \& Hirata, C. 2012, arXiv:1201.1005

Walsh, S. M., Jerjen, H., \& Willman, B. 2007, ApJ, 662, L83

Whalen, D., O'Shea, B. W., Smidt, J., \& Norman, M. L. 2008, ApJ, 679, 925

Willman, B., et al. 2005, ApJ, 626, L85

Xue, X. X., et al. 2008, ApJ, 684, 1143

York, D. G., et al. 2000, AJ, 120, 1579

Zahn, O., et al. 2011, ApJ, submitted, arXiv:1111.6386

Zucker, D. B., et al. 2006, ApJ, 650, L41 